\title{
氮杂 Brazilin/二芳基狮杂合物的合成研究
}

\author{
潘成学 ${ }^{a, b}$ 关一富 ${ }^{a}$ 张洪涁*, $a$ \\ ( ${ }^{a}$ 云南大学化学科学与工程学院 教育部自然资源药物化学重点实验室 昆明 650091) \\ $\left({ }^{b}\right.$ 广西师范大学化学化工学院 药用资源化学与药物分子工程国家重点实验室培育基地 桂林 541004)
}

\begin{abstract}
摘要 天然产物在药物化学中具有非常重要的地位, 天然产物杂合物的设计合成, 可以为药物篎选提供数目更多、结 构更多样化的生物活性分子, 是发现更多的新药先导化合物的一条非常重要的途径. 以邻苯二甲醚为原料, 经傅克酰 基化、酮酯缩合、Knoevenagel 缩合、Nazarov 环化、酮酸酯的胺解、1,3-二美基化合物的 $\alpha$-羊基化、酮羰基和酰胺的 还原及分子内的傅克环化共 9 步反应, 合成了氮杂 brazilin (Aza-brazilin)与 1,3-二芳基苑类化合物的一个杂合物.
\end{abstract}

关键词＼cjkstart氮杂 brazilin; 天然产物杂合物; 1,3-二芳基狮; 合成

\section{Synthesis of Aza-brazilin/diarylindan-Based Hybrid}

\author{
Pan, Chengxue Guan, Yifu $^{a} \quad$ Zhang, Hongbin ${ }^{*, a}$
}

$\left({ }^{a}\right.$ Key Laboratory of Medicinal Chemistry for Natural Resource, Ministry of Education, Yunnan University, Kunming 650091) ( ${ }^{b}$ State Key Laboratory Cultivation Base for the Chemistry and Molecular Engineering of Medicinal Resources,

Guangxi Normal University, Guilin 541004)

\begin{abstract}
Natural products play the most important role in the development of drugs. The design and synthesis of natural products hybrids seem to be a promising approach to increase the number and the diversity of compounds for pharmacological testing and were expected to disclose an avenue toward the development of valuable leads for medicinal applications. In this paper the synthesis of an aza-brazilin/1,3-diarylindan-based hybrid starting from 1,2-dimethoxy-benzene was reported. The synthesis finished in 9 steps including Friedel-Crafts acylation, Claisen condensation, Knoevenagel condensation, Nazarov cyclization, aminolysis of $\beta$-keto esters, $\alpha$-hydroxylation of $\beta$-dicarbonyl compounds, reduction of ketone, amide and the intra-molecular Friedel-Crafts cyclization reaction.
\end{abstract}

Keywords aza-brazilin; natural products hybrid; 1,3-diarylindan; synthesis

天然产物是各种药物先导化合物的重要源泉, 从活 性天然产物骨架中去寻找新药及新药研发信息, 是新药 研发的最重要途径 ${ }^{1]}$. 天然产物杂合物(Natural product hybrids)的设计合成, 是 20 世纪末 21 世纪初开始出现的 一种从天然产物骨架中去寻找先导化合物的新途径, 其 基本的策略是将不同天然产物的生物活性结构单元与 活性分子或药物分子之间进行有机的组合来形成新的 化合物, 以达到优化、提高单一的天然产物本身的生物 活性，或者从中发现新的活性化合物的目的 ${ }^{[2,3]}$.

天然产物杂合物的设计合成可以用 Tietze 课题组 ${ }^{[4]}$ 早前报道的一个比较典型的例子来说明. 他们以天然产 物 1 (Estrone)和 2 (Talaromycin)为基础, 设计合成出了 这两个化合物的杂合物 3 . 活性研究结果发现,

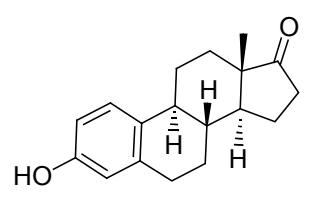

1

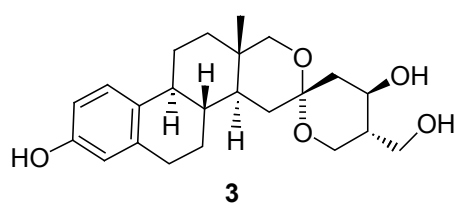

Hydrib of 1 and 2<smiles>CC[C@H]1CO[C@]2(CO1)C[C@H](O)[C@@H](CO)CO2</smiles>

2
目标化合物显示很好的抗肿瘤活性, 其对多种癌细胞的 选择性及抑制活性均比母体化合物有了显著的提高.

\footnotetext{
*E-mail: zhanghb@ynu.edu.cn; panling74@163.com

Received September 4, 2011; revised March 1, 2012; published online March 18, 2012.

Project supported by the National Basic Research Program of China (973 Program, No. 2009CB522300).

国家 973 重大基础研究计划子项目(No. 2009CB522300)资助项目.
} 
目前, 天然产物杂合物的设计理念开始受到广泛关

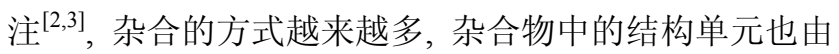
最初的以天然产物为主, 拓展到药物及其它的活性分 子, 为活性分子的发现与䇥选提供了数目巨大且结构非 常多样化的候选分子. 被认为是寻找各种生物活性分 子、药物先导化合物以及其他功能分子的一个前景光 明、充满希望的新方向.

(+)-Brazilin 是从药材苏方木中分离得到的天然产 物, 具有抗菌 ${ }^{[5]}$ 、抗炎 ${ }^{[6]}$ 、降低血液粘度 ${ }^{[7]}$ 、抑制端粒酶 活性 ${ }^{[8]}$ 及切割 $\mathrm{DNA}^{[9]}$ 等, 其衍生物的合成方法及活性研 究也因此引起了许多课题组的兴趣 ${ }^{[10 \sim 12]}$. 最近, 我们报 道了氮杂 brazilin $4 \mathrm{~b}$ 及其一些衍生物的合成, 发现他们 具有较好的抗肿瘤活性 ${ }^{[13]}$. 受天然产物杂合物的设计 理念, 以及 Cousins 等 ${ }^{[14]}$ 报道的内皮素受体拮抗剂 (EDNR Antagonist) 5 的合成与活性研究工作的启发, 我 们设计了 $4 \mathrm{~b}$ 与 5 的杂合物 6 的合成, 以研究其生物活性 及其与母体化合物活性的相互联系和差别, 具体的合成 路线见 Scheme 1.

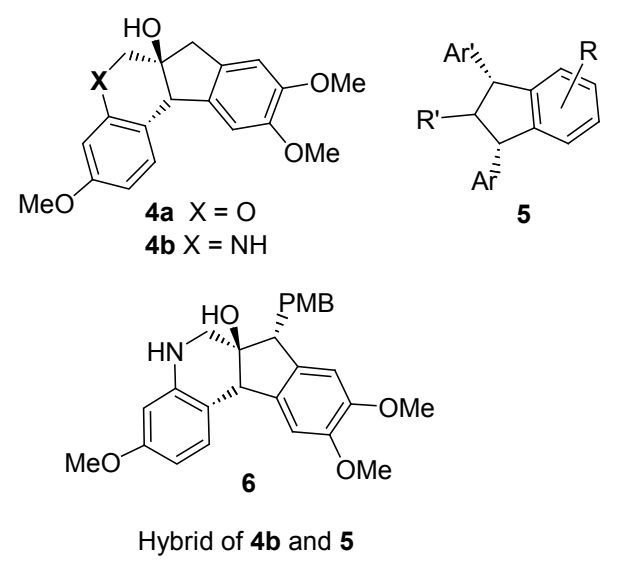

\section{1 实验部分}

\section{1 仪器与试剂}

Bruker Avance 300 核磁共振仪(TMS 为内标, 德国 布鲁克公司); BRUER HTC 型离子阱质谱仪(德国布鲁 克公司); Vario Micro cube 元素分析仪(德国 elementar 公 司); XT-4 型双目显微熔点仪(北京泰克仪器设备有限公 司); 薄层层析及柱层析所用硅胶板及硅胶均为中国青 岛海洋化工集团公司生产. 所用无水溶剂均按照标准方 法严格处理, 使用前蒸出. 原料间甲氧基苯胺为 Acros 产品; 其它试剂均为国产试剂, 除特别说明外, 均是购 买后直接使用.

\subsection{1-(3,4-二甲氧苯基)乙酮(8)的合成}

将醋酐 $(10 \mathrm{~mL}, 100 \mathrm{mmol})$ 溶解在无水二氯甲烷(150
$\mathrm{mL})$ 中, 加入无水三氯化铝 $(13.5 \mathrm{~g}, 100 \mathrm{mmol})$, 在室温 下搅拌 1 2 min 后, 再往其中加入邻苯二甲醚 7 (6.9 g, $50 \mathrm{mmol}$ ), 在室温下搅拌约 $1 \mathrm{~h}$, 然后小心分批加入适量 冰块停止反应. 粗产物用二氯甲烷萃取后用饱和氯化钠 溶液洗涤, 无水硫酸钠干燥. 过滤, 减压除去溶剂, 用 硅胶柱层析提纯[展开剂: $V$ (乙酸乙酯) : $V$ (石油醚) $=$ $1: 10$ ] 得到 $5.4 \mathrm{~g}$ 淡黄色液体 8 , 产率 $60 \% .{ }^{1} \mathrm{H}$ NMR $\left(\mathrm{CDCl}_{3}, 300 \mathrm{MHz}\right) \delta: 7.56(\mathrm{dd}, J=8.4,1.8 \mathrm{~Hz}, 1 \mathrm{H}, \mathrm{ArH})$, $7.51(\mathrm{~d}, J=1.8 \mathrm{~Hz}, 1 \mathrm{H}, \mathrm{ArH}), 6.88(\mathrm{~d}, J=8.4 \mathrm{~Hz}, 1 \mathrm{H}$, $\mathrm{ArH}), 3.93$ (s, 3H, $\left.\mathrm{OCH}_{3}\right), 3.92$ (s, $\left.3 \mathrm{H}, \mathrm{OCH}_{3}\right), 2.55$ (s, $3 \mathrm{H}$, $\left.\mathrm{COCH}_{3}\right) ;{ }^{13} \mathrm{C}$ NMR $\left(\mathrm{CDCl}_{3}, 75 \mathrm{MHz}\right) \delta: 196.4,153.1$, 148.8, 130.2, 123.1, 109.9, 109.8, 55.8, 55.7, 25.9 .

\subsection{3-(3,4-二甲氧苯基)-3-氧代-丙酸甲酯(9)的合成}

将芳基乙酮 $8(9.0 \mathrm{~g}, 50 \mathrm{mmol})$ 溶解在无水四氢呋喃 (THF) $(150 \mathrm{~mL})$ 中, 加入碳酸二甲酯 $(27 \mathrm{~g}, 0.3 \mathrm{~mol})$, 然 后再小心加入 $60 \%$ 的氢化钠 $(4.0 \mathrm{~g}, 100 \mathrm{mmol})$, 在氮气 保护下回流 2 3 h. 冷却至室温后, 在冰水浴冷却下用 稀盐酸小心中和至中性. 粗产物用乙酸乙酯萃取, 饱和 氯化钠溶液洗涤, 无水硫酸钠干燥. 过滤, 减压除去溶 剂, 用硅胶柱层析提纯[展开剂: $V$ (乙酸乙酯) : $V$ (石油 醚) $=1: 4$ ] 得到 $8.9 \mathrm{~g}$ 浅黄色液体 9, 产率 75\%. ${ }^{1} \mathrm{H} \mathrm{NMR}$ $\left(\mathrm{CDCl}_{3}, 300 \mathrm{MHz}\right) \delta: 7.48 \sim 7.54(\mathrm{~m}, 2 \mathrm{H}, \mathrm{ArH}), 6.89(\mathrm{~d}$, $J=8.4 \mathrm{~Hz}, 1 \mathrm{H}, \mathrm{ArH}), 3.98\left(\mathrm{~s}, 2 \mathrm{H}, \mathrm{CH}_{2}\right), 3.92(\mathrm{~s}, 3 \mathrm{H}$, $\left.\mathrm{OCH}_{3}\right), 3.89$ (s, $\left.3 \mathrm{H}, \mathrm{OCH}_{3}\right), 3.72\left(\mathrm{~s}, 3 \mathrm{H}, \mathrm{OCH}_{3}\right) ;{ }^{13} \mathrm{C} \mathrm{NMR}$ $\left(\mathrm{CDCl}_{3}, 75 \mathrm{MHz}\right) \delta: 190.5,167.7,153.4,148.6,128.6$, 123.1, 109.7, 55.5, 55.3, 51.8, 44.8 .

\subsection{2-(3,4-二甲氧苯甲酰基)-3-(4-甲氧苯基)丙烯酸甲 酯(10)的合成}

将芳基酮酸酯 9 (7.2 g, $30 \mathrm{mmol}$ ) 和对甲氧基苯甲 醛 $(4.9 \mathrm{~g}, 33 \mathrm{mmol})$ 溶解在苯 $(150 \mathrm{~mL})$ 中, 加入 $0.5 \mathrm{~mL}$ 六 氢吡啶, 在氮气保护下回流分水 $6 \mathrm{~h}$. 待反应完全后, 减 压除去溶剂, 粗产物用乙酸乙酯萃取; 然后依次用稀盐 酸、饱和氯化钠溶液洗涤, 无水硫酸钠干燥. 过滤, 减压 除去溶剂, 用乙酸乙酯-石油醚重结晶, 得 $8.1 \mathrm{~g}$ 淡黄色 固体 10, 产率 76\%. m.p. $171 \sim 173{ }^{\circ} \mathrm{C} ;{ }^{1} \mathrm{H} \mathrm{NMR}\left(\mathrm{CDCl}_{3}\right.$, $300 \mathrm{MHz}) \delta: 7.90(\mathrm{~s}, 1 \mathrm{H}, \mathrm{CH}), 7.63(\mathrm{~d}, J=1.5 \mathrm{~Hz}, 1 \mathrm{H}$, $\mathrm{ArH}), 7.50$ (dd, $J=8.6,1.2 \mathrm{~Hz}, 1 \mathrm{H}, \mathrm{ArH}), 7.32$ (d, $J=8.9$ $\mathrm{Hz}, 2 \mathrm{H}, \mathrm{ArH}), 6.80$ (dd, $J=8.6,1.2 \mathrm{~Hz}, 1 \mathrm{H}, \mathrm{ArH}), 7.32$ (d, $J=8.9 \mathrm{~Hz}, 2 \mathrm{H}, \mathrm{ArH}), 3.93$ (s, $\left.3 \mathrm{H}, \mathrm{OCH}_{3}\right), 3.89$ (s, $3 \mathrm{H}$, $\left.\mathrm{OCH}_{3}\right), 3.74\left(\mathrm{~s}, 3 \mathrm{H}, \mathrm{OCH}_{3}\right), 3.72\left(\mathrm{~s}, 3 \mathrm{H}, \mathrm{OCH}_{3}\right) ;{ }^{13} \mathrm{C} \mathrm{NMR}$ $\left(\mathrm{CDCl}_{3}, 75 \mathrm{MHz}\right) \delta: 194.5,165.9,161.3,154.1,149.4$, $142.1,132.2,129.3,128.1,125.4,125.0,114.2,110.3$, $110.2,56.0,55.9,55.2,52.4$. 

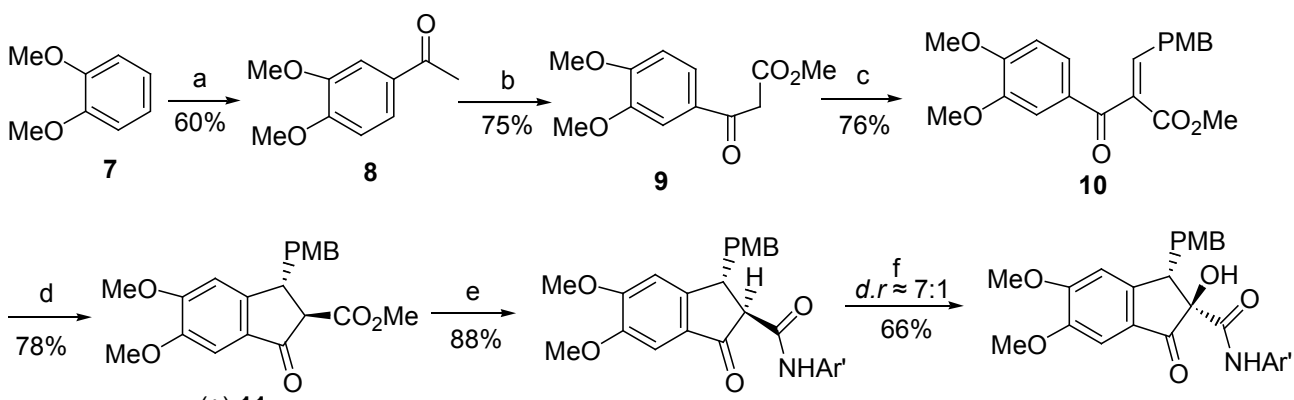

$( \pm) 11$

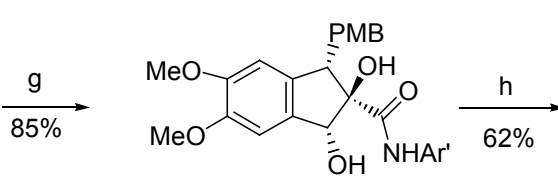

( \pm ) 14
( \pm ) 12

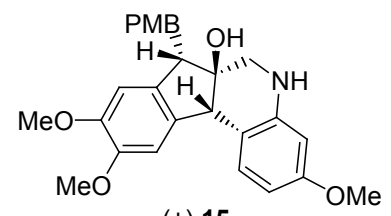

( \pm$) 15$
( \pm ) 13

Reagents and conditions: (a) $\mathrm{Ac}_{2} \mathrm{O}$ (2 equiv.), $\mathrm{AlCl}_{3}$ (2 equiv.)/DCM, r.t.; (b) ( $\left.\mathrm{MeO}\right)_{2} \mathrm{CO}$ (6 equiv.), $\mathrm{NaH}$ (2 equiv.), reflux; (c) PMBCHO (1.1 equiv.)/Benzene, Piperidine (0.5 mL), reflux; (d) $\mathrm{BF}_{3}-\mathrm{Et}_{2} \mathrm{O}$ (3 equiv.)/DCM, $0{ }^{\circ} \mathrm{C} \sim$ r.t.; (e) $\mathrm{Ar}^{\prime} \mathrm{NH}_{2}(2$ equiv.)/xylene, reflux; (f) $\mathrm{O}_{2}(101 \mathrm{kPa}) / V(D M F): V\left(i\right.$-propanol) $=1: 1, \mathrm{CeCl}_{3}-7 \mathrm{H}_{2} \mathrm{O}$ (0.5 equiv.), r.t.; (g) $\mathrm{NaBH}_{4}$ (3 equiv.), $\mathrm{MeOH}, 0^{\circ} \mathrm{C} \sim$ r.t.; (h) (i) $\mathrm{LAH}(3$ equiv. $) / \mathrm{THF}, 0^{\circ} \mathrm{C} \sim \operatorname{reflux}$; (ii) $\mathrm{BF}_{3} \mathrm{Et}_{2} \mathrm{O}(5$ equiv. $) / \mathrm{DCM}, 0{ }^{\circ} \mathrm{C}$

\section{Scheme 1}

\subsection{5,6-二甲氧基-1-(4-甲氧苯基)-3-氧代狮-2-甲酸甲} 酯(11)的合成

将化合物 10 (7.1 g, $20 \mathrm{mmol})$ 溶解在无水二氯甲烷 $(150 \mathrm{~mL})$ 中, 在 $0{ }^{\circ} \mathrm{C}$ 下逐滴加入 $45 \% \sim 47 \%$ 的三氟化硼 乙醚 $(16.5 \mathrm{~mL}, 60 \mathrm{mmol})$, 然后在室温下继续摚拌 $1 \sim 2$ $\mathrm{h}$. 反应完成后, 在冰水浴冷却下小心往烧瓶中加小块 碎冰, 然后加入饱和碳酸氢钠溶液 $(50 \mathrm{~mL})$, 在室温下摚 拌 $30 \mathrm{~min}$. 分出有机层, 水层用二氯甲烷萃取(150 $\mathrm{mL} \times 2)$, 合并有机层, 用饱和食盐水洗涤 2 次, 无水硫 酸钠干燥. 过滤, 减压除去溶剂, 用硅胶柱层析提纯展 开剂[展开剂: $V$ (二氯甲烷) $: V$ (石油醚) $=1 ： 1$ ] 得到 5.5 $\mathrm{g}$ 淡黄色固体 11, 产率 78\%. m.p. 131 132 ${ }^{\circ} \mathrm{C} ;{ }^{1} \mathrm{H}$ NMR $\left(\mathrm{CDCl}_{3}, 300 \mathrm{MHz}\right) \delta: 7.17$ (s, 1H, ArH), 7.03 (d, $J=8.0$ $\mathrm{Hz}, 2 \mathrm{H}, \mathrm{ArH}), 6.82$ (d, $J=8.0 \mathrm{~Hz}, 2 \mathrm{H}, \mathrm{ArH}), 6.61$ (s, 1H, $\mathrm{ArH}), 4.82(\mathrm{~d}, J=3.9 \mathrm{~Hz}, 1 \mathrm{H}, \mathrm{ArCH}), 3.88\left(\mathrm{~s}, 3 \mathrm{H}, \mathrm{OCH}_{3}\right)$, $3.83\left(\mathrm{~s}, 3 \mathrm{H}, \mathrm{OCH}_{3}\right), 3.74\left(\mathrm{~s}, 3 \mathrm{H}, \mathrm{OCH}_{3}\right), 3.73(\mathrm{~s}, 3 \mathrm{H}$, $\left.\mathrm{OCH}_{3}\right), 3.57(\mathrm{~d}, J=3.9 \mathrm{~Hz}, 1 \mathrm{H}, \mathrm{CH}) ;{ }^{13} \mathrm{C} \mathrm{NMR}\left(\mathrm{CDCl}_{3}, 75\right.$ MHz) $\delta: 197.0,169.2,158.9,156.4,152.1,150.2,133.9$ $128.9,127.9,114.4,107.3,104.2,63.8,56.3,56.2,55.3$, $52.7,47.7$.

\section{$1.6 \mathrm{~N}$-3-甲氧苯基-5,6-二甲氧基-1-(4-甲氧苯基)-3-氧} 代狮-2-甲酰胺(12)的合成

将化合物 11 (5.3 g, $15.0 \mathrm{mmol}$ )溶解在二甲苯 (50 $\mathrm{mL})$ 中, 再加入间甲氧基苯胺 $(3.7 \mathrm{~g}, 30 \mathrm{mmol})$, 在氮气 保护下回流 $2.5 \sim 3.5 \mathrm{~h}$; 反应完成后, 减压除去溶剂, 粗 产物用硅胶柱层析提纯展开剂[展开剂: $V$ (乙酸乙酯)：
$V($ 石油醚 $)=1 ： 2$ 得 $5.9 \mathrm{~g}$ 白色固体 12, 产率 $88 \%$. m.p. $120 \sim 122{ }^{\circ} \mathrm{C} ;{ }^{1} \mathrm{H}$ NMR $\left(\mathrm{CDCl}_{3}, 300 \mathrm{MHz}\right) \delta: 9.12(\mathrm{~s}, 1 \mathrm{H}$, $\mathrm{NH}), 7.06 \sim 7.30(\mathrm{~m}, 6 \mathrm{H}, \operatorname{ArH}), 6.85(\mathrm{~d}, J=8.4 \mathrm{~Hz}, 2 \mathrm{H}$, $\operatorname{ArH}), 6.68(\mathrm{~s}, 1 \mathrm{H}, \operatorname{ArH}), 6.64(\mathrm{~d}, J=8.1 \mathrm{~Hz}, 1 \mathrm{H}, \operatorname{ArH})$, $5.15(\mathrm{~d}, \quad J=3.0 \mathrm{~Hz}, 1 \mathrm{H}, \mathrm{ArCH}), 3.93\left(\mathrm{~s}, 3 \mathrm{H}, \mathrm{OCH}_{3}\right), 3.86$ (s, $\left.3 \mathrm{H}, \mathrm{OCH}_{3}\right), 3.78\left(\mathrm{~s}, 6 \mathrm{H}, \mathrm{OCH}_{3}\right), 3.57(\mathrm{~d}, J=3.0 \mathrm{~Hz}$, $1 \mathrm{H}, \mathrm{CH}) ;{ }^{13} \mathrm{C}$ NMR $\left(\mathrm{CDCl}_{3}, 75 \mathrm{MHz}\right) \delta: 200.7,164.4$, $160.2,158.8,157.0,153.1,150.2,139.1,134.8,129.6$, $129.2,127.8,114.4,112.2,110.4,107.6,105.5,104.0$, $64.1,56.5,56.2,55.3,45.7$.

\section{$1.7 \mathrm{~N}$-3-甲氧苯基-5,6-二甲氧基-1-(4-甲氧苯基)-2-羟 基-3-氧代狮-2-甲酰胺(13)的合成}

将化合物 12 (4.8 g, $10 \mathrm{mmol}$ )溶解在异丙醇-DMF $(50 \mathrm{~mL}+50 \mathrm{~mL})$ 混合溶液中, 再加入 $1.5 \mathrm{~mL}$ 水及 0.5 equiv.的七水合三氯化铈, 在室温下于 $101 \mathrm{kPa}$ 氧气中搅 拌反应 $48 \mathrm{~h}$. 反应完全后, 减压除去大部分溶剂, 用乙 酸乙酯萃取, 无水硫酸钠干燥. 过滤, 减压除去溶剂, 用硅胶柱层析提纯 [展开剂: $V$ (乙酸乙酯) : $V$ (石油醚 $)=$ $1: 2$ ]得到 $3.1 \mathrm{~g}$ 白色固体 13, 产率 66\%. m.p. 178 179 ${ }^{\circ} \mathrm{C} ;{ }^{1} \mathrm{H}$ NMR $\left(\mathrm{CDCl}_{3}, 300 \mathrm{MHz}\right) \delta: 8.31(\mathrm{~s}, 1 \mathrm{H}, \mathrm{NH})$, $7.23 \sim 7.27(\mathrm{~m}, 3 \mathrm{H}, \mathrm{ArH}), 6.98(\mathrm{~d}, J=8.1 \mathrm{~Hz}, 1 \mathrm{H}, \mathrm{ArH})$, $6.72 \sim 6.80(\mathrm{~m}, 4 \mathrm{H}, \mathrm{ArH}), 6.49 \sim 6.55(\mathrm{~m}, 2 \mathrm{H}, \mathrm{ArH}), 5.07$ (s, $1 \mathrm{H}, \mathrm{ArCH}), 4.56(\mathrm{~s}, 1 \mathrm{H}, \mathrm{OH}), 3.88\left(\mathrm{~s}, 3 \mathrm{H}, \mathrm{OCH}_{3}\right), 3.85$ (s, $\left.3 \mathrm{H}, \mathrm{OCH}_{3}\right), 3.69\left(\mathrm{~s}, 3 \mathrm{H}, \mathrm{OCH}_{3}\right), 3.63\left(\mathrm{~s}, 3 \mathrm{H}, \mathrm{OCH}_{3}\right)$;

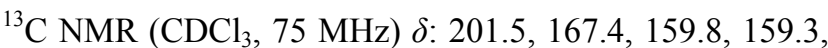
$156.7,150.2,149.7,137.7,130.9,129.2,128.1,113.8$, $112.5,110.9,107.2,105.7,104.7,88.2,57.0,56.5,56.2$, 
55.2, 55.2; APCI-MS $m / z: 464.32(\mathrm{M}+1)^{+}$. Anal. calcd for $\mathrm{C}_{26} \mathrm{H}_{25} \mathrm{NO}_{7} \mathrm{C}$ 67.38, H 5.44, N 3.02; found C 67.39, H 5.21, N 2.91.

\section{$1.8 \mathrm{~N}$-3-甲氧苯基-5,6-二甲氧基-1-(4-甲氧苯基)-2,3- 二羟基狮-2-甲酰胺(14)的合成}

将化合物 13 (5.0 mmol) 溶解在 THF-甲醇 $(100 \mathrm{~mL}+$ $10 \mathrm{~mL}$ )混合液中, 在冰水浴冷却下分批加入嗍氢化钠 $(0.57 \mathrm{~g}, 15 \mathrm{mmol})$, 在室温下搅拌 $2 \mathrm{~h}$; 加入饱和氯化铵 溶液 $(20 \mathrm{~mL})$ 和乙酸乙酯 $(50 \mathrm{~mL})$, 分出有机层, 水层用 乙酸乙酯 $(50 \mathrm{~mL} \times 4)$ 萃取, 无水硫酸钠干燥. 过滤, 减 压除去溶剂, 粗产物用乙酸乙酯重结晶得 $1.94 \mathrm{~g}$ 白色固 体 14, 产率 85\%. m.p. $116 \sim 118{ }^{\circ} \mathrm{C} ;{ }^{1} \mathrm{H}$ NMR $\left(\mathrm{CDCl}_{3}\right.$, $300 \mathrm{MHz}) \delta: 8.87(\mathrm{~s}, 1 \mathrm{H}, \mathrm{NH}), 7.14(\mathrm{~d}, J=8.6 \mathrm{~Hz}, 2 \mathrm{H}$, ArH), $6.95 \sim 7.00(\mathrm{~m}, 2 \mathrm{H}, \operatorname{ArH}), 6.73(\mathrm{~d}, J=8.6 \mathrm{~Hz}, 2 \mathrm{H}$, ArH), $6.64(\mathrm{~d}, J=1.8 \mathrm{~Hz}, 1 \mathrm{H}, \operatorname{ArH}), 6.44 \sim 6.54(\mathrm{~m}, 3 \mathrm{H}$, ArH), 5.29 (s, 1H, CHOH), 4.42 (s, 1H, ArCH), 3.85 (s, $\left.3 \mathrm{H}, \mathrm{OCH}_{3}\right), 3.72\left(\mathrm{~s}, 3 \mathrm{H}, \mathrm{OCH}_{3}\right), 3.66\left(\mathrm{~s}, 3 \mathrm{H}, \mathrm{OCH}_{3}\right), 3.62$ (s, $\left.3 \mathrm{H}, \mathrm{OCH}_{3}\right) ;{ }^{13} \mathrm{C} \mathrm{NMR}\left(\mathrm{CDCl}_{3}, 75 \mathrm{MHz}\right) \delta: 170.4$, $159.7,159.1,149.8,149.5,137.4,134.3,131.9,130.2$, $129.5,129.2,113.8,112.9,110.7,107.7,106.2,106.0$, 91.9, 82.5, 59.2, 56.1, 56.0, 55.2, 55.1; APCI-MS $m / z$ : $466.67(\mathrm{M}+1)^{+}$. Anal. calcd for $\mathrm{C}_{26} \mathrm{H}_{27} \mathrm{NO}_{7} \mathrm{C} 67.09, \mathrm{H}$ 5.85, N 3.01; found C 67.13, H 5.77, N 3.20.

\section{$1.93,9,10$-三甲氧基-7-(4-甲氧苯基)-5,6,7,11-四氢狮 [2,1-c]喹啉-6-醇(15)的合成}

将化合物 14 (2.0 mmol) 溶解在无水 THF $(50 \mathrm{~mL})$ 中, 在冰水浴下加入氢化铝锂 $(0.23 \mathrm{~g}, 6 \mathrm{mmol})$, 在室温和氮 气氛中搅拌反应 $1 \mathrm{~h}$, 然后加热回流 $6 \mathrm{~h}$. 在冰水浴冷却 下小心加入 $30 \mathrm{~mL}$ 饱和氯化铵溶液, 然后再加入水(20 $\mathrm{mL}$ )和乙酸乙酯 $(50 \mathrm{~mL})$. 分出有机层, 水层再用乙酸乙 酯萃取 $(50 \mathrm{~mL} \times 3)$. 合并有机层, 用饱和食盐水洗涤两 次, 无水硫酸钠干燥. 过滤, 减压除去溶剂, 将所得粗 产物溶解于无水二氯甲烷 $(80 \mathrm{~mL})$ 中, 在冰水浴冷却下 加入 $45 \% \sim 47 \%$ 的三氟化嗍乙醚 $(2.8 \mathrm{~mL}, 10 \mathrm{mmol})$, 继 续搅拌约 $1 \mathrm{~h}$; 小心往其中加入饱和碳酸氢钠溶液 $(20$ $\mathrm{mL}$ ), 在室温下继续搅拌 $1 \mathrm{~h}$. 分出有机层, 水层用二氯 甲烷萃取 $(75 \mathrm{~mL} \times 2)$; 合并有机层, 用饱和食盐水洗涤 两次, 无水硫酸钠干燥. 过滤, 减压除去溶剂, 用硅胶 柱层析提纯 [展开剂: $V$ (乙酸乙酯) $: V$ (石油醚) $=1: 1$ ] 得 到 $0.54 \mathrm{~g}$ 浅紫色晶体 15, 产率 62\%. m.p. $227 \sim 228{ }^{\circ} \mathrm{C}$; ${ }^{1} \mathrm{H} \mathrm{NMR}\left(\mathrm{CDCl}_{3}, 300 \mathrm{MHz}\right) \delta$ : $7.21 \sim 7.29$ (m, 3H, ArH), 6.91 (d, $J=8.4 \mathrm{~Hz}, 2 \mathrm{H}, \operatorname{ArH}), 6.80$ (s, 1H, ArH), 6.67 (s, 1H, ArH), 6.48 (dd, $J=8.4,2.1 \mathrm{~Hz}, 1 \mathrm{H}, \mathrm{ArH}), 6.14$ (d, $J=$ $2.1 \mathrm{~Hz}, 1 \mathrm{H}, \mathrm{ArH}), 5.78(\mathrm{~s}, 1 \mathrm{H}, \mathrm{ArH}), 4.68(\mathrm{~s}, 1 \mathrm{H}, \mathrm{ArCH})$, $4.08(\mathrm{~s}, 1 \mathrm{H}, \mathrm{ArCH}), 3.82\left(\mathrm{~s}, 3 \mathrm{H}, \mathrm{OCH}_{3}\right), 3.81(\mathrm{~s}, 3 \mathrm{H}$,
$\left.\mathrm{OCH}_{3}\right), 3.77$ (s, 3H, $\left.\mathrm{OCH}_{3}\right), 3.74\left(\mathrm{~s}, 3 \mathrm{H}, \mathrm{OCH}_{3}\right), 2.87$ (d, $\left.J=14.6 \mathrm{~Hz}, 1 \mathrm{H}, \mathrm{CH}_{\mathrm{a}} \mathrm{H}\right), 2.23\left(\mathrm{~d}, J=14.6 \mathrm{~Hz}, 1 \mathrm{H}, \mathrm{CHH}_{\mathrm{b}}\right.$ ); ${ }^{13} \mathrm{C} \mathrm{NMR}\left(\mathrm{CDCl}_{3}, 75 \mathrm{MHz}\right) \delta: 159.2,158.9,148.6,148.4$, $144.9,137.7,132.5,132.0,130.8,129.2,113.9,113.8$, 109.2, 107.4, 105.2, 100.1, 80.8, 57.0, 56.3, 56.2, 55.3, 55.2, 51.5, 45.8; APCI-MS $m / z: 434.86(\mathrm{M}+1)^{+}$. Anal. calcd for $\mathrm{C}_{26} \mathrm{H}_{27} \mathrm{NO}_{5} \mathrm{C} 72.04, \mathrm{H} 6.28, \mathrm{~N} 3.23$; found $\mathrm{C}$ 72.46, H 5.97, N 3.36 .

\section{2 结果与讨论}

由芳醚 7 经傅克酰基化反应制备芳乙酮 8 , 我们分 别尝试了乙酰氯和醋酲两种不同的酰化剂, 发现以乙酰 氯为酰化剂时转化率较高且反应速度较快, 但产物中有 脱甲醚的芳乙酮生成, 分离提纯很困难, 所以我们最终 采用醋䣶作为酰化剂.

缩合产物 $\mathbf{1 0}$ 的 Nazarov 环化，文献报道的一些类似 类反应一般是用三氟甲磺酸、甲磺酸等质子酸或三氯化 铝等路易斯酸为催化剂 ${ }^{[15]}$. 但化合物 10 在以上催化剂 下进行环化反应, 产物都比较复杂. 考虑到这个底物的 芳环都比较活泼, 太强的酸会使反应过于剧烈而导致产 物复杂化, 最后我们尝试了以三氟化嗍为催化剂, 终于 以比较理想的收率得到了环化产物 $\mathbf{1 1}$.

从胺解产物 12 的活泼次甲基中氧化出一个羟基, 我们主要是参考 Christoffers 等报道的 $\beta$-二酮或酮酸酯 的 $\alpha$-羟基化的方法 ${ }^{[16]}$. 文献采用的反应溶剂是异丙醇, 由于 12 在异丙醇中溶解度不好, 所以以异丙醇为溶剂 时反应没能发生, 为此我们尝试用异丙醇与 DCM, THF 及 DMF 等的混合溶剂, 发现在异丙醇与 DMF 的混合溶 剂中反应可以顺利进行. 羟基化反应中可得到的两个非 对映体,经柱层析分离后, 确定其比例大约为 $7: 1$. 从文 献 ${ }^{[16]}$ 报道的反应机理来推断, 水分子主要是从位阻较 小的一边去进攻 $\mathrm{Ce}^{4+}$ 氧化出来的正离子, 所以产物以 为 trans 构型为主 (Scheme 2).
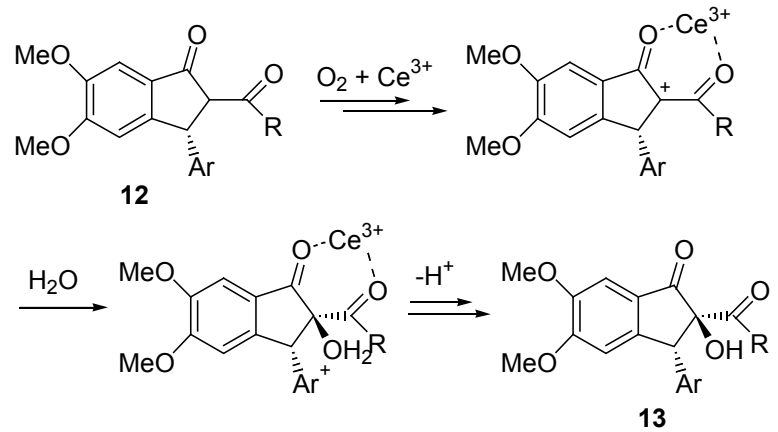

Scheme 2

13 的还原成 14 的转化具有很好的立体选择性, 反 应只分离得到一个非对映体产物. 其可能的原因是, 底 
物 13 中与羟基同侧的一边位阻较小, 而且还原剂 $\mathrm{NaBH}_{4}$ 容易与羟基氧结合, 导致其主要是从与羟基同侧 的一面进攻酮羰基，从而得到两个羟基为反式构型的还 原产物 14.14 再经过常规的氢化铝锂还原进而再进行分 子内傅克环化反应, 即可得到目标产物 15.15 的二维 NOE 谱图显示两个茮位上的氢之间具有强的 NOE 效应, 说明这两个氢处于五元环的同侧. 结合化合物 13 的立 体化学可以推断, 目标产物 15 中不仅狮环与六元环的 并环方式与天然产物 brazilin 相同, 且狮环上的两个芳 基呈顺式,即与具有内皮素受体抑制活性的 1,3-二芳基 䒢类化合物的构型相同.

\section{3 结论}

我们以邻苯二甲醚为原料, 经 9 步反应, 设计合成 了一个氮杂 brazilin 与 1,3 -二芳基狮的杂合物. 杂合物保 留了天然产物 brazilin 的相对构型, 其狮环上的两个芳 基也保留了具有内皮素受体抑制活性的 1,3-二芳基茚类 化合物的顺式构型. 目前这个化合物的生物活性正在研 究中.

\section{References}

[1] (a) Newman, D. J.; Cragg, G. M. J. Nat. Prod. 2007, 70, 461.

(b) Newman, D. J.; Cragg, G. M. Future Med. Chem. 2009, 1, 1415 .

[2] For reviews, see: (a) Gademann, K. Chimia 2006, 60, 841.

(b) Tietze, L. F.; Bell, H. P.; Chandrasekhar, S. Angew. Chem., Int. Ed. 2003, 42, 3996.

(c) Mehta, G.; Singh, V. Chem. Soc. Rev. 2002, 31, 324.

[3] for selected examples, see: (a) Alvaro, E.; de la Torre, M. C.; Si- erra, M. A. Chem. Eur. J. 2006, 12, 6403.

(b) Wach, J. Y.; Bonazzi, S.; Gademann, K. Angew. Chem., Int. Ed. 2008, 47, 7123.

[4] (a) Tietze, L. F.; Schneider, G.; Wolfling, J.; Fecher, A.; Nobel, T.; Petersen, S.; Schuberth, I.; Wulff, C. Chem. Eur. J. 2000, 6, 375.

(b) Tietze, L. F.; Schneider, G.; Wolfling, J.; Nbel, T.; Wulff, C.; Schuberth, I.; Rbeling, A. Angew. Chem., Int. Ed. 1998, 37, 2469.

[5] Pratt, R.; Yuzuriha, Y. J. Am. Pharm. Assoc. 1959, 48, 69.

[6] Bae, I. K.; Min, H. Y.; Han, A. R.; Seo, E. K.; Lee, S. K. Eur. J. Pharmacol. 2005, 513, 237.

[7] Moon, C. K.; Chung, J. H.; Lee, Y. M.; Lee, S. H.; Hwang, G. S.; Park, K. S.; Mock, M. S.; Kim, S. G.; Ahn,Y. S.; Ann, J. H. Arch. Pharmacol. Res. 1988, 11, 149.

[8] Tolman, R. L.; Chin, A. C. WO 0193864, 2001 [Chem. Abstr. 2001, 136, 15230].

[9] Mar, W.; Lee, H. T.; Je, K. H.; Choi, H. Y.; Seo, E. K. A. Arch. Pharmacol. Res. 2003, 26, 147.

[10] Davis, F. A.; Chen, B. C. J. Org. Chem. 1993, 58, 1751.

[11] Huang, Y. D.; Zhang, J. S.; Pettus, T. R. R. Org. Lett. 2005, 7, 5841.

[12] Yen, C. T.; Goto, K. N.; Hwang, T. L; Wu, P. C.; Natschke, S. M.; Lai, W. C.; Bastowd, K. F.; Chang, F. R.; Wu, Y. C.; Lee, K. H. Bioorg. Med. Chem. Lett. 2010, 20, 1037.

[13] Pan, C. X.; Zeng, X. H.; Guan, Y. F.; Jiang, X. L.; Li, L.; Zhang, H. B. Synlett 2011, 425

[14] (a) Cousins, R. D.; Elliott, J. D.; Lago, M. A.; Leber, J. D.; Peishoff, C. E. US 5817693, 1998 [Chem. Abstr. 1998, 129, 289938]. (b) Elliott, J. D.; Lago, M. A.; Cousins, R. D.; Gao, A.; Leber, J. D.; Erhard, K. F.; Nambi, P.; Elshourbagy, N. A.; Kumar, C. J. Med. Chem. 1994, 37, 1553.

[15] (a) Kerr, D. J.; Metje, C.; Flynn, B. L. Chem. Commun. 2003, 12, 1380 .

(b) Kerr, D. J.; White, J. M.; Flynn, B. L. J. Org. Chem. 2010, 75, 7073.

[16] Christoffers, J.; Werner, T.; Unger, S.; Frey, W. Eur. J. Org. Chem. 2003, 425 . 